\title{
El impacto en los primeros años de aplicación del programa Oportunidades por tipo de pobreza en México y Baja California, 2002-2006*
}

\section{The impact in the early years of the Oportunidades program by type of poverty in Mexico and Baja California, 2002-2006 Joaquín Bracamontes Nevárez ${ }^{* *}$, Mario Camberos Castro** y Luis Huesca Reynoso**}

Resumen

En este trabajo se muestra el limitado impacto del programa Oportunidades en la reducción de la pobreza en México y Baja California durante el periodo de estudio 2002-2006, lo que obedece a la insuficiencia de recursos y la exclusión de hogares pobres. En el país los tres tipos de pobreza tuvieron una modesta reducción y en Baja California la pobreza de capacidades disminuyó ligeramente, pero la pobreza alimentaria y la patrimonial se incrementaron pese a Oportunidades.

Palabras clave: líneas de pobreza, mocrosimulación estática, pobreza.

\begin{abstract}
This paper shows a limited impact of the Oportunidades program in reducing poverty in Mexico and Baja California during the study period (2002-2006), which is due to insufficient resources and the exclusion of poor households. In the country the three types of poverty had a modest reduction and in Baja California capabilities poverty declined slightly, but the food and heritage poverty increased despite the program.

Keywords: poverty lines, static microsimulation, poverty.
\end{abstract}

Recibido el 26 de febrero de 2013.

Aprobado el 25 de abril de 2014.

* Luis Huesca agradece al Conacyt por su apoyo en la modalidad de Estancia sabática en el extranjero para consolidación de grupos (ES1-10010-2013-01) en la Université Laval, Département d'économique, Quebec, Canadá, donde concluyó su parte correspondiente a esta investigación.

** Profesores-investigadores del Centro de Investigación en Alimentación y Desarrollo, A. C. (CIAD), Departamento de Economía, Coordinación de Desarrollo Regional.Correos electrónicos: joaco@ciad.mx,mcamberos@ciad.mx, lhuesca@ciad.mx 


\section{Introducción}

$\mathrm{Al}$ iniciar el siglo xxi la pobreza constituye un problema de primer orden a escala mundial y se torna el principal objetivo de desarrollo en el milenio, ${ }^{1}$ lo que llevó a establecer la meta de reducirla a la mitad para el año 2015. En el mundo había alrededor de tres mil millones de personas que vivían en pobreza con menos de dos dólares americanos per cápita diarios (World Bank, 2005), de los cuales 200 millones habitaban en América Latina (Perry, Arias, López, Maloney y Servén, 2006) y más de 50 millones en México (Banco Mundial, 2004; Damian y Boltvinik, 2003; Sedesol-ctmp, 2002).

En este contexto, en marzo de 2002 se crea el programa Oportunidades para combatir la pobreza ${ }^{2}$ en el país, el cual asocia la pobreza a las restricciones económicas que enfrentan los individuos en el acceso a los mínimos indispensables para desarrollar sus capacidades básicas: una buena nutrición, salud y educación e insertarse de esa forma en la vida social y productiva (Sedesol, 2003). Por ello, mediante este programa se otorgan transferencias monetarias a las familias buscando elevar las capacidades básicas de las personas que conforman los hogares en pobreza.

No obstante, aunque Oportunidades significa una gran ayuda para las familias que no satisfacen los mínimos de bienestar, surgen preguntas como las siguientes: ¿En verdad el programa Oportunidades ha jugado un papel importante para reducir la pobreza en México?, ¿qué tanto ha contribuido este programa para la reducción de los niveles de pobreza en Baja California?, ¿̇es suficiente el monto monetario mensual que el

${ }^{1}$ En la ciudad de Nueva York, en septiembre de 2000, los 189 estados miembros de las Naciones Unidas adoptaron las Ocho Metas del Milenio para ser alcanzadas en 2015: 1) erradicación de la pobreza extrema y el hambre; 2) acceso universal a la educación primaria; 3) promover la igualdad de géneros; 4) reducción de la mortalidad infantil; 5) mejorar la salud materna; 6) combatir el viH/sida y otras enfermedades; 7) asegurar la sostenibilidad medioambiental; y 8 ) desarrollar asociaciones globales. Al respecto, véase $<$ www.un.org/es/millenniumgoals/ $>$.

${ }^{2}$ El programa Oportunidades cuenta con la mayor asignación presupuestal que haya tenido el rubro de combate a la pobreza en cualquier programa federal y gradualmente se le ha incrementado el recurso asignado: pasó de 22 mil millones de pesos en el 2002 a 33 mil millones en el 2006, y a alrededor de 60 mil millones para el 2010; véase para cada año el Decreto de Presupuesto de Egresos de la Federación. 
programa ofrece a los hogares para combatir de manera efectiva la pobreza en el país y en Baja California?

El propósito en este trabajo es conocer el impacto del programa Oportunidades en el combate a la pobreza en el país y en el estado fronterizo de Baja California en el periodo 2002-2006. Por ello se proponen tres objetivos específicos: 1) estimar los niveles de pobreza en México y en Baja California para los años 2002 y 2006; 2) evaluar el impacto del programa con la Técnica de Microsimulación Estática; y 3) estimar si las transferencias monetarias de Oportunidades coadyuvan al combate efectivo de la pobreza en el país y en Baja California.

Es importante advertir que el estado de Baja California - junto con Nuevo León- se ha caracterizado por tener los niveles más bajos de pobreza en la Frontera Norte de México ${ }^{3}$ (Aguilar, 2000; Camberos y Bracamontes, 1996), lo cual sugiere la relevancia de valorar el papel y la pertinencia que puede tener el programa Oportunidades en la erradicación de la pobreza. La hipótesis a probar es que las transferencias monetarias que otorga el programa Oportunidades han coadyuvado a reducir de manera significativa los niveles de pobreza en el país y también en Baja California durante el periodo 2002-2006.

Luego de esta sección introductoria, el trabajo se organiza en seis secciones más. La segunda sección incluye la conceptualización de la pobreza, la tercera aborda la explicación metodológica y la cuarta describe la evaluación de la política social en el país. En la quinta sección se analiza el impacto del programa Oportunidades en México y Baja California; luego, la sexta sección hace algunas consideraciones sobre los costos económicos en el combate a la pobreza y los recursos destinados al programa Oportunidades en el país y en Baja California. En la última sección se presentan las conclusiones.

\section{La conceptualización de la pobreza}

Este estudio se fundamenta en el "enfoque monetario de la pobreza" o paradigma de la pobreza de ingresos, el cual tiene su origen en los esfuerzos

${ }^{3}$ Integrada por las seis entidades que tienen colindancia con Estados Unidos de América: Baja California, Sonora, Chihuahua, Coahuila, Nuevo León y Tamaulipas. 
pioneros de Benjamín S. Rowntree a inicios del siglo xx. Este paradigma, se centra en los ingresos o gastos para medir la pobreza ${ }^{4}$ y naturalmente conduce a la estrategia del crecimiento en el ingreso nacional para la reducción de ésta (Kanbur y Squire, 2001).

Dicho enfoque supone que las diferencias en el bienestar de la población se pueden resumir por las diferencias en los ingresos o gastos de consumo; es decir, los hogares/individuos pobres se definen por la condición de insuficiencia de ingresos para cubrir el costo de determinados satisfactores básicos. Entonces, dependiendo de la unidad de análisis, todos los hogares o personas transforman los ingresos en bienestar de igual manera (Bourguignon, 2003).

Por lo tanto, los pobres son definidos por la condición de que sus ingresos caen por debajo de una línea de pobreza arbitrariamente predeterminada. Esta línea de pobreza puede definirse en términos absolutos, como lo hace el Banco Mundial para las comparaciones internacionales de la pobreza con 1 o 2 dólares de los Estados Unidos por persona al día, y también en términos relativos considerando un porcentaje de la media de ingresos de la población.

El supuesto clave es que con herramientas debidamente elaboradas las cifras monetarias uniformes pueden tomar en cuenta la heterogeneidad en la situación de los hogares y las personas. Este enfoque propició un gran trabajo durante el siglo pasado y subyace en buena parte de la literatura económica sobre pobreza de los últimos 30 años (Bourguignon, 2003; Kanbur, 2002), por lo que se le considera dominante.

\section{El método de líneas de pobreza, la técnica de microsimulación estática y análisis no paramétrico}

\section{El método de líneas de pobreza}

Primero se emplea el método de líneas de pobreza (LP) y los índices FGT (Sen, 1976; Foster, Greer y Thoerbecke, 1984) para estimar los niveles de

${ }^{4}$ En el transcurso del siglo pasado este enfoque se replanteó y fue desarrollado de manera formal por Sen (1976). 
pobreza, luego se recurre a la técnica de microsimulación estática (TMSE) para medir el impacto del programa Oportunidades en la reducción de la pobreza (Bourguignon y Spadaro, 2006). El método de líneas de pobreza toma como referencia el costo de los satisfactores básicos o mínimos llamado línea de pobreza $(Z)^{5}$, por lo que se consideran en pobreza aquellas familias o individuos cuyo ingreso $\left(Y_{i}\right)^{6}$ está por debajo de la línea de pobreza $(Z)$. Entonces, si se cumple la condición $\left(Z-Y_{i}\right)>0$, el hogar será pobre $\left(n_{i}\right)$ y el total de hogares en pobreza será $\left(N_{i}\right)$.

Los índices de pobreza FGT de Foster, Greer y Thorbecke (1984), introducen un ponderador " $\alpha$ " que es un factor de valoración subjetiva del grado de pobreza. Cuando el parámetro $\alpha=0$, el índice $F G T(0)$ es el porcentaje de hogares en pobreza; mientras que, si $\alpha=1$ el índice FGT (1) representa la intensidad promedio de la pobreza; es decir, cuanto mayor es el índice $F G T$ (1), en promedio los ingresos de los hogares pobres estarán más alejados de las líneas de pobreza.

En atención a los axiomas de pobreza de Sen $(1976)^{7}$, Foster y colaboradores (1984) utilizan el parámetro $\alpha=2$ para medir la "severidad" de la pobreza; es decir, el índice $F G T$ (2) refleja la mayor sensibilidad al déficit de ingreso de los hogares más pobres o aversión a la pobreza. La fórmula generalizada de los índices FGT es la siguiente:

$$
F G T(\alpha)=P_{\alpha}(y, z)=\frac{1}{n} \sum_{i=1}^{i=q}\left(\frac{z-y_{i}}{z}\right)^{\alpha}
$$

${ }^{5}$ Véase el desarrollo formal del método de líneas de pobreza en Camberos, Genesta y Huesca (1996, pp. 163-200).

${ }^{6}$ Es el ingreso neto total per cápita mensual de los hogares el que se compara con el valor monetario de las líneas de pobreza; es decir, el ingreso familiar, luego de pagar impuestos y recibir transferencias del gobierno.

${ }^{7}$ Amartya Sen menciona dos axiomas deseables para un índice de pobreza. Un primer axioma es el de monotonicidad: si lo demás se mantiene constante, una reducción en el ingreso de un individuo por debajo de la línea de pobreza debe incrementar el índice de pobreza. El segundo axioma es el de transferencia: si lo demás se mantiene constante, una transferencia de ingreso de una persona pobre a otra más rica debe incrementar el índice de pobreza (Sen, 1976). 
Donde $n$ es el número total de hogares observados, $q$ el número de hogares pobres, $y_{i}$ el ingreso per cápita de los hogares pobres, $z$ representa las líneas de pobreza y $\alpha$ el parámetro de ponderación del grado de pobreza en los hogares o de los individuos $(\alpha=0,1$ y 2$)$.

\section{La técnica de microsimulación estática}

Las técnicas de microsimulación son un instrumento del análisis económico que permite estudiar los efectos que tiene un cambio de la política fiscal y social en la calidad de vida de la población (Bourguignon y Spadaro, 2006). Esta técnica se torna muy importante porque ayuda a modelar/ simular la política social, dando respuesta a la siguiente pregunta: ¿Cuál sería el nivel de pobreza ante una cambio de la variable $Y_{i}$ para el hogar h en el tiempo $t+1$ si se aplica determinada política social y todo lo demás se mantiene constante?

En términos generales, la técnica de microsimulación estática presenta las siguientes características: 1) se utilizan microdatos con información detallada de los hogares individuos; 2) se suponen constantes tanto la estructura demográfica como la económica; 3) no se considera la reacción de los agentes económicos ante un cambio de política; y 4) por tanto, se trata de cálculos que muestran el efecto inmediato de un cambio en la política fiscal o social (Cogneau, Grimm y Robillird, 2003).

\section{Los softwares utilizados y microsimulación de la política social}

Para estimar niveles de pobreza se sigue la metodología propuesta por el Comité Técnico para la Medición de la Pobreza (стмP) (Sedesol, 2002); la fuente de información es la Encuesta Nacional de Ingresos y Gastos de los Hogares (ENIGH) (2002 y 2006) del Instituto Nacional de Estadística y Geografía (INEGI). Se utiliza el software DAD 4.4 (DAD: A Software for Poverty and Distributive Análisis ${ }^{8}$, uno de los más apropiados en el análisis de la

${ }^{8}$ El DAD es uno de los software de que dispone el Banco Mundial para el análisis y monitoreo de la pobreza, programado en lenguaje Java (Duclos y Araar, 2006). 
pobreza y la distribución del ingreso (Zhan, 2003). Para ello, a partir de los microdatos de las ENIGH, se estimaron los niveles de ingreso neto total per cápita mensual familiar mediante el paquete estadístico STATA.

Los creadores del DAD, Duclos y Araar (2006), advierten tres factores que motivaron su construcción: 1) la necesidad de que los investigadores en los países en desarrollo realicen análisis propios sobre pobreza y desigualdad; 2) la accesibilidad a microdatos debido al mayor almacenamiento y la velocidad de procesamiento propiciado por la revolución informática; y 3) el hecho de que gran parte del análisis de distribución era manejado como si no estuviera sujeto a imprecisión estadística. Por tanto, el DAD fue diseñado para coadyuvar a que el análisis distributivo ofrezca resultados estadísticamente significativos.

La microsimulación de la política social se hace de la manera siguiente: Primero, se calcula la pobreza con el método de LP, considerando el ingreso per cápita mensual de los hogares que incluye todas las fuentes de ingreso y Oportunidades, obteniendo así los índices de pobreza existentes en México y Baja California; segundo, se restan las transferencias de ingreso que el programa Oportunidades hace al ingreso per cápita mensual del hogar y se microsimula un mundo sin transferencias, el cual será punto de referencia para comparar el impacto del programa; la diferencia entre ellos es el impacto de Oportunidades en el combate a la pobreza. Tercero, se calcula la pobreza microsimulando la transferencia focalizada del monto máximo en apoyo al ingreso per cápita mensual de todo hogar en situación de pobreza. ${ }^{9}$

El programa Oportunidades establece un monto monetario máximo que se puede transferir a las familias en pobreza, pero el otorgamiento de este monto depende de las particularidades de cada hogar; es decir, en realidad a los hogares se les da el apoyo monetario para alimentos y energía, pero el incremento de las transferencias está sujeto a que el hogar tenga hijos en educación básica o media superior y a la existencia o no de adultos mayores en la familia. ${ }^{10}$ Entonces, bajo estas consideraciones, el

${ }^{9}$ De acuerdo con las cifras históricas de los apoyos, en 2002 el monto máximo de apoyo mensual por familia era de 1550 pesos (1.29 salarios mínimos mensuales) y 1855 pesos (1.31 Smm) para el 2006; véase <www.oportunidades.gob.mx/Portal/wb/ Web/oportunidades_historico>.

${ }^{10}$ Véase las reglas de operación del programa en <www.oportunidades.gob.mx>. 
apoyo monetario que el programa Oportunidades otorga a los hogares en realidad no sólo es focalizado también es un apoyo dosificado. ${ }^{11}$

\section{El análisis no paramétrico}

Para tener una visión de conjunto de los cambios en la pobreza que producen las transferencias monetarias de programas como el de Oportunidades, se analiza la información mediante las funciones de distribución del ingreso familiar con la herramienta de tipo no paramétrico kernel (Silverman, 1986), al mismo tiempo que se calcula la proporción de la densidad de la pobreza para así examinar el impacto de Oportunidades en el país y las regiones antes y después de haber recibido las transferencias del programa mencionado.

La técnica de medición requiere obtener las distribuciones de las transferencias, que en este caso son las recibidas por el programa Oportunidades, y un escenario de tipo universal para todos los pobres. Procedemos con la estimación de las densidades de los ingresos de las familias que permite observar el patrón de comportamiento en las distintas funciones de distribución de probabilidad (FDP). Así, las densidades son estimadas con la técnica no paramétrica kernel, al utilizar una muestra que denominaremos $j$ en lugar de la población total.

Se calcula una función de densidad $\hat{f}(x)$ sobre un vector de ingresos $x=\left(x_{1}, \ldots, x_{n}\right)$ bajo el supuesto de que la muestra para los individuos caracterizados por $i$ ha sido extraída de su propia densidad de población original $f\left(x_{j}\right)$, obteniendo así la forma real aproximada de la distribución de los ingresos de la densidad poblacional. En nuestro caso, el estimador común se aplica sobre el ingreso familiar per cápita y se calcula de manera no paramétrica como lo indica la expresión (2), donde para toda $j$ tenemos:

$$
\hat{f}\left(x_{j}\right)=\frac{1}{h} \sum_{i=1}^{n} K\left(\frac{x_{j}-x_{i}}{h}\right) \forall j
$$

${ }^{11}$ En este sentido, de acuerdo con Hernández y Del Razo (2004, pp. 31-32), los hogares beneficiarios del programa recibían en promedio 370 pesos por mes, equivalentes a 26\% del ingreso familiar en el año 2003. 
Siendo $h$ la banda óptima y una constante $K$ como función kernel de tipo gaussiano. Se sugiere la banda óptima ya que una mayor puede sobresuavizar la densidad estimada; es por ello que en este trabajo se estima la óptima y representativa para cada distribución, como lo sugiere Silverman (1986) y Wand y Jones (1995). Se emplea un estimador kernel adaptativo (Jenkins y Van Kerm, 2005) que mantiene una reducida variabilidad de los puntos estimados en donde la información de los datos es escasa, permitiendo distinguir posibles modas que comúnmente se ocultan al emplear un estimador kernel tradicional.

Para no estimar funciones de los ingresos que puedan estar sobre-representando las densidades de sus distribuciones, y por tanto, poder contar con una descomposición de los subgrupos de las simulaciones de la pobreza patrimonial, se calculan sus densidades tomando en cuenta el peso de su grupo. El procedimiento se realiza multiplicando su peso relativo respectivo con cada transferencia simulada del programa (antes, después y la transferencia universal con $K$ grupos $=1,2,3$ ) de la siguiente manera:

$$
f(x)=\sum_{k=1}^{j} v^{k} f^{k}(x)
$$

La función de densidad $f(x)$ para cada nivel de ingreso $x$ es una suma ponderada de la distribución de pobreza para cada subgrupo $k$ de transferencia específica, donde $v^{k}$ es la participación relativa del subgrupo de pobres $k \mathrm{y} f^{k}$ es su propia FDP, lo que permite sumar un área igual al porcentaje relativo de la pobreza total estimada en cada tipo de transferencia correspondiente a cada escenario.

\section{Evaluación de la política social en México}

En México, en términos de la política pública, hasta ahora la preocupación central en la implementación de los diferentes programas sociales ha sido la cobertura, el ejercicio de los recursos y a veces el costo que implica la puesta en práctica de los mismos, actividades que se inscriben en el ámbito de la evaluación de metas y en menor medida en relación con el impacto social que pudieran tener los diferentes programas sociales (González, 2002). 
De esta manera, Bautista (2004) estudia el efecto de Oportunidades sobre la utilización de servicios básicos de salud durante el periodo 19962002, en las clínicas de primer nivel de atención por parte de los hogares beneficiarios del medio rural. Encontró que la demanda por consultas totales en las familias incorporadas a Oportunidades ha crecido más en el periodo analizado respecto a la demanda de las familias no incorporadas. En las clínicas incorporadas se requieren en promedio más de dos consultas más por día, que en las no incorporadas.

Además, los resultados del análisis indican que las consultas de nutrición crecen significativamente más rápido en las comunidades que pertenecen al programa, pero no parece haber un efecto muy fuerte sobre la disminución de consultas a niños con desnutrición severa. Por tanto, Oportunidades ha mostrado su efectividad en elevar de manera constante la demanda de servicios de básicos de salud en las comunidades rurales. La expectativa es que el aumento en la utilización de servicios se refleje en una mejoría en los niveles de salud de la población.

Parker (2003) evalúa la inscripción de nivel primaria, secundaria y media superior para las zonas rurales, semiurbanas y urbanas. Se analiza la inscripción a nivel de escuela antes y después de la implementación del programa, las escuelas se clasifican como Oportunidades (aquellas en las que había al menos un becario) y escuelas no Oportunidades (aquellas en donde no había becarios). Los estimadores son de dobles diferencias, se comparan los cambios en la inscripción en las escuelas Oportunidades a lo largo del tiempo con cambios en la inscripción en las escuelas no Oportunidades.

No se observa impacto del programa en la inscripción primaria de las zonas rurales y urbanas, las secundarias rurales registran importantes aumentos en la matrícula lo que no ocurre en las secundarias rurales - quizá por la reducida cobertura en estas zonas hasta el 2001—; mientras que en secundarias semiurbanas los aumentos se dan en la matrícula femenina. En las escuelas de nivel medio superior, tanto en zonas rurales, semiurbanas y urbanas el impacto se dio principalmente en el primer grado, lo cual no sorprende pues es el primer año en que el programa se implementa a este nivel; sin embargo, en conjunto los incrementos en la matrícula sugieren que Oportunidades está aumentando fuertemente los niveles de escolaridad de su población beneficiaria. 
Hernández y Del Razo (2004) evalúan el impacto de los programas sociales a partir de la percepción de los beneficiarios. Utilizan la base de datos de la encuesta Lo que Dicen los Pobres de la Secretaría de Desarrollo Social (Sedesol, 2003), que capta información de las características individuales y familiares de una muestra representativa a escala nacional de familias en pobreza. Utilizando la metodología de propensity score matching (apareamiento por propensión), construyen un grupo contrafactual de individuos que no reciben programas sociales para comparar sus percepciones con la de aquellos individuos que sí son beneficiarios.

Estos autores encontraron: a) el conjunto de individuos que reciben programas sociales percibe que tiene un mejor nivel económico y social que el grupo contrafactual que no recibe beneficios; $b$ ) quienes no reciben programas sociales tienen que llevar a cabo un esfuerzo laboral adicional, especialmente de los niños y otros miembros que no son el jefe de la familia; c) los individuos que reciben programas tienen una mejor percepción de las acciones del gobierno; y $d$ ) entre los beneficiarios se puede percibir un sentimiento de dependencia del gobierno, así como de impotencia para poder solucionar los problemas por ellos mismos, lo cual no ocurre entre los individuos que no reciben programas sociales.

Por su parte, Cortés, Bánegas y Solís (2007) estudian y hacen una comparación con análisis ex-ante y ex-post de los recursos de Oportunidades por zona rural y urbana. Estos autores encuentran que en los años 2002 y 2005, los recursos del programa Oportunidades han tenido efecto reductor de la pobreza en el entorno rural, cuando no ha sido así para el caso urbano, lo que en buena medida está en línea con los hallazgos en este trabajo para México y Baja California.

La evaluación de la política pública no ha puesto atención al estudio y evaluación de la política social con base en las técnicas de microsimulación; además, la mayoría de los estudios realizados hasta hoy se inscribe más bien en la evaluación de metas del programa Oportunidades más que en la evaluación de su impacto social. En este sentido, auxiliados en la técnica de microsimulación estática, el interés en este trabajo consiste en cuantificar el impacto de las transferencias monetarias que ofrece el programa Oportunidades a las familias mexicanas en relación con los niveles de pobreza en México y Baja California para el periodo 2002-2006. 


\section{Las líneas de pobreza, funciones de distribución del ingreso y densidad de la pobreza: Incidencia e impacto del programa Oportunidades}

En este trabajo se aplican las líneas de pobreza obtenidas a partir de la canasta alimentaria INEGI-CEPAL (citado en Sedesol, 2002), actualizadas según los desagregados por rubro y bienes específicos del Índice Nacional de Precios al Consumidor, que son replicadas luego por el Consejo Nacional de Evaluación de la Política de Desarrollo Social (Coneval, 2006). No es suficiente una canasta básica alimentaria para construir las líneas de pobreza, también se deben considerar otras necesidades básicas no alimentarias; para ello, el procedimiento más común y el cual se emplea en el estudio INEGI-CEPAL (1993), consiste en aplicar al valor monetario de la canasta alimentaria un factor de expansión que es el inverso del coeficiente de Engel, ${ }^{12}$ para estimar así cada línea de pobreza.

\section{Líneas de pobreza}

En el cuadro 1 se pueden ver las líneas de pobreza ${ }^{13}$ calculadas por el Coneval, las cuales se utilizan en este trabajo para estimar los niveles de pobreza y en la microsimulación de la política social. En el año 2002 todo hogar cuyo ingreso neto total per cápita mensual fuese inferior o igual a 672.27 pesos —equivalentes a 0.56 salarios mínimos mensuales (SMM) se consideraba que estaba en pobreza alimentaria; mientras que todo hogar con un ingreso neto total per cápita mensual igual o inferior a 824.54

${ }^{12}$ El economista prusiano Engel, observa una tendencia entre el presupuesto destinado a alimentos y el gasto total, señalando que cuando aumenta el ingreso, la proporción que se gasta en alimentos disminuye - Ley de Engel-. Para ver como el cтмP y el Coneval estiman el coeficiente de Engel y lo utilizan en la estimación de las Líneas de Pobreza, véase Sedesol (2002) en el Anexo 1.

${ }^{13}$ La LP alimentaria es el valor o costo monetario de la canasta básica alimentaria InegiCepal (1993) la cual provee los mínimos requerimientos nutricionales por persona al mes, la LP de capacidades comprende el costo monetario de la canasta básica alimentaria, salud y educación; mientras que, la LP de patrimonio implica el costo monetario por adquirir la canasta básica alimentaria, salud, educación, vestido, calzado, vivienda y transporte público por persona al mes. 
pesos (0.69 SMm) se consideraba en pobreza de capacidades; y los hogares que tuvieran un ingreso neto total per cápita mensual de 1348.84 pesos (1.12 Sмm) o menos, estaban en pobreza de patrimonio.

Cuadro 1. México. Líneas de pobrezaa mensual per cápita, 2002 y 2006

\begin{tabular}{|lcccc|}
\hline \multicolumn{1}{|c}{ Tipo de Pobreza } & Año 2002 & S.M.M. ${ }^{b}$ & Año 2006 & S.M.M. \\
\hline Alimentaria & 672.27 & 0.56 & 809.87 & 0.57 \\
De Capacidades & 824.54 & 0.69 & 993.31 & 0.70 \\
De Patrimonio & 1348.84 & 1.12 & 1624.92 & 1.15 \\
\hline
\end{tabular}

a Ingreso neto mensual per cápita en pesos de agosto para cada año.

${ }^{\text {b }}$ Es la equivalencia en Salarios Mínimos Mensuales.El SMM en 2002 era \$1203.00 pesos y en el $2006 \$ 1,414.80$ pesos; véase www.conasami.gob.mx

Fuente. Tomado del coneval que hace la estimación siguiendo la metodología del ctmp y la información de los deflactores necesarios proporcionada por el Banco de México, véase Nota Técnica 001 del CONEVAL 2006 y 2007.

Para el año 2006, los hogares que tuvieran un ingreso neto total per cápita mensual igual o menor a 809.87 pesos (0.57 SMm) se consideraba que estaban en condición de pobreza alimentaria. Padecían pobreza de capacidades los hogares con un ingreso neto total per cápita mensual igual o por debajo de 993.31 pesos (0.70 SMm); mientras que se consideraban pobres de patrimonio los hogares cuyo ingreso neto total per cápita mensual era igual o menor a 1624.92 (1.15 SMm).

Funciones de distribución del ingreso y densidad de la pobreza: Antes y después del programa Oportunidades

Para una valoración de conjunto de los cambios que produce la política pública y en particular el programa Oportunidades en la pobreza, es importante analizar la información mediante las funciones de distribución del ingreso familiar con la herramienta kernel (Silverman, 1986) y la proporción de la densidad de la pobreza, para analizar el impacto del programa en el país y el estado antes y después de las transferencias de Oportunidades, 
así como los efectos en la distribución del ingreso de los hogares pobres en el supuesto de que se transfiriera el monto máximo ${ }^{14}$ a todos los hogares pobres - transferencia universal—, efectos que en el apartado siguiente serán mejor precisados.

Figura 1. Función de la densidad del ingreso y escenarios de transferencias en México, 2006

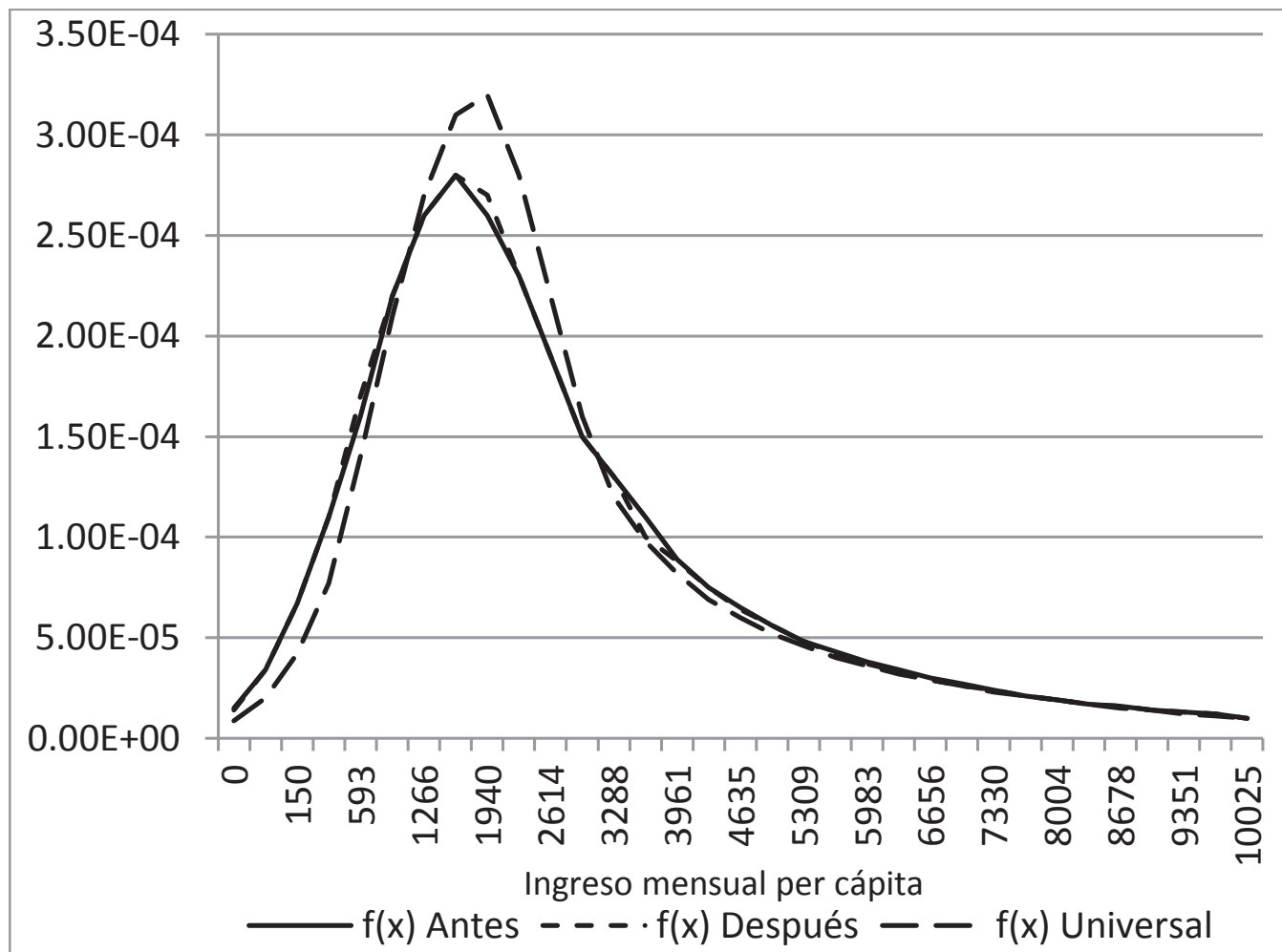

En México se puede ver cómo las funciones de distribución de los ingresos antes (curva continua) y después (curva segmentos pequeños) de Oportunidades no se modifican sustancialmente y el cambio operado después del

${ }^{14}$ Como se ha señalado en el apartado metodológico, si bien el programa Oportunidades establece un monto monetario máximo que se puede transferir a las familias en pobreza, en realidad a los hogares se les da el apoyo monetario para alimentos y energía, pero el incremento de dicho monto está sujeto a que el hogar tenga hijos en educación básica o media superior y a la existencia o no de adultos mayores en la familia; véase las reglas de operación del programa en <www.oportunidades.gob.mx>. 
subsidio apenas incrementa la moda y en la parte baja de ambas distribuciones los cambios son imperceptibles (figura 1). En contraste, se ve de manera contundente que la distribución de los ingresos con una transferencia universal (curva segmentos grandes) a todos los hogares detectados como pobres induce una mejora sustancial y muy superior que las transferencias de Oportunidades en el país. Hay un desplazamiento uniforme hacia la derecha desde los más pobres (por debajo del umbral alimentario), lo que significaría reducir la situación de pobreza a una proporción mucho menor de hogares.

Para Baja California (figura 2), la función de distribución presenta una moda más elevada y situada ligeramente hacia la derecha respecto de la función de distribución del país, señal de menor desigualdad y concentración de hogares en esa parte de la distribución; lo cual muestra que una menor proporción de grupos de ingreso medio se ubicó por debajo de los tres tipos de pobreza - líneas verticales- en Baja California. Cuando se incluye Oportunidades, se observa un efecto positivo en tanto

Figura 2. Función de la densidad del ingreso y escenarios de transferencias en Baja California, 2006

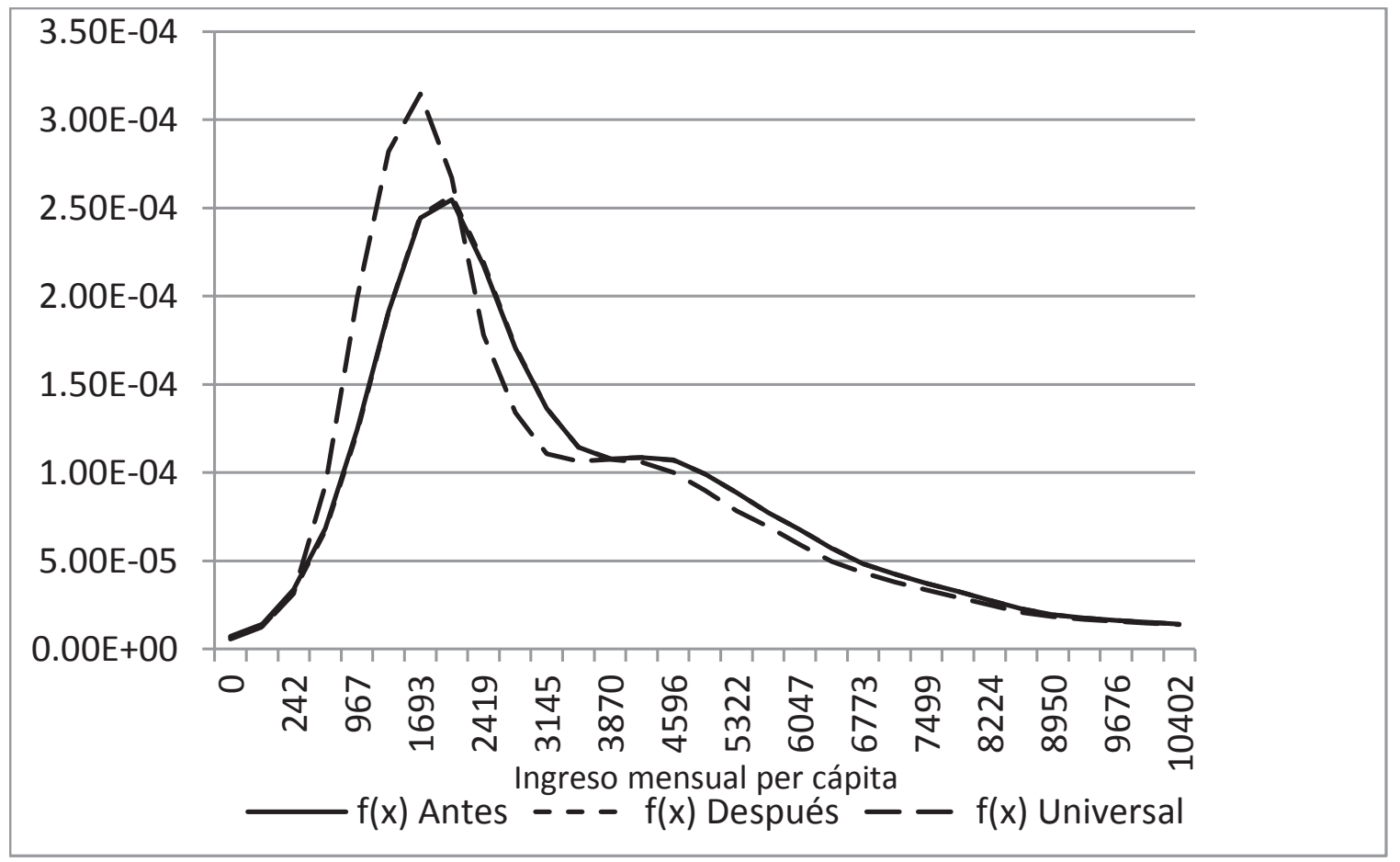


que traslada la curva distributiva hacia la derecha y de manera apenas perceptible aleja a los grupos medios de los tres umbrales de pobreza, aunque en menor medida de la patrimonial, como se prueba adelante más detalladamente.

Un efecto similar pero más intenso se consigue en el umbral de pobreza alimentaria con la transferencia universal, efecto que "aparentemente" no se observa en los umbrales de pobreza de capacidades y patrimonial - lo que reflejaría la inclusión de hogares no pobres en el ejercicio de focalización- ${ }^{15}$ pero que se confirma de mejor manera al analizar la densidad de la pobreza para Baja California.

En México (figura 3), en términos de la función de densidad de la pobreza total (patrimonial) - considerando solamente la densidad de los pobres-, se observa que Oportunidades tiene un impacto más notable solamente en la pobreza alimentaria y por debajo de 680 pesos per cápita y muy reducido por encima de dicho umbral. Destacan los incrementos de la pobreza que no aparecían en las funciones de distribución del ingreso que se ubican en los estratos por encima de los 680 y hasta los 820 pesos y superior de los 1300 hasta los 1600 pesos (línea segmentos pequeños) en el 2006. En estos estratos la densidad de la distribución de pobres, después de Oportunidades, se ubica por encima de la misma antes de transferencias, señal de exclusión a hogares que requerían el apoyo y no se les otorgó. Igual sucede si observamos con mayor precisión en los tramos iniciales de la densidad, donde se ubica la pobreza alimentaria, específicamente en el tramo que va de los 380 hasta los 420 pesos per cápita. Por otra parte, las funciones de densidad de la pobreza (suponiendo la transferencia del monto máximo) muestran que en el país se tendría un impacto mucho mayor que el observado en la actualidad por el programa.

${ }^{15}$ Los autores Cornia y Stewart (2003) advierten sobre la existencia de dos errores en la focalización de los programas que transfieren apoyos monetarios, como es el caso del programa Oportunidades, a saber: el error de omisión o exclusión de los pobres (error tipo I) y el error de inclusión de los no pobres (errores tipo II), lo que a la postre tiene que ver con la eficiencia y eficacia en la implementación de dichos programas. Para efecto de constatar la gravedad de este tipo de problemática en el país véase a Bracamontes, Camberos y Huesca (2011). 
Figura 3. México, función de la densidad de los ingresos en pobreza de patrimonio y escenarios de transferencias en 2006

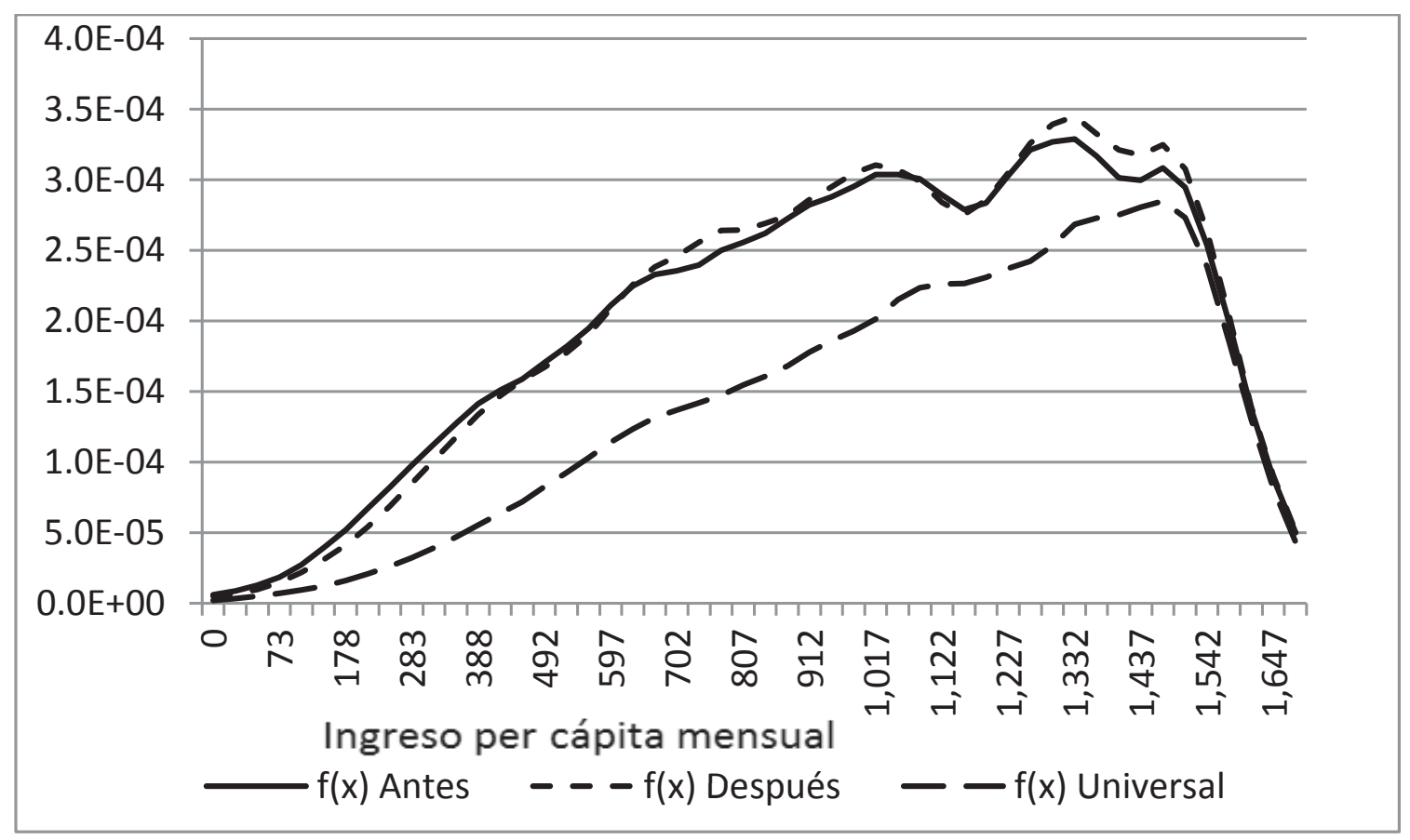

Figura 4. Baja California, función de la densidad de los ingresos en pobreza de patrimonio y escenarios de transferencias en 2006

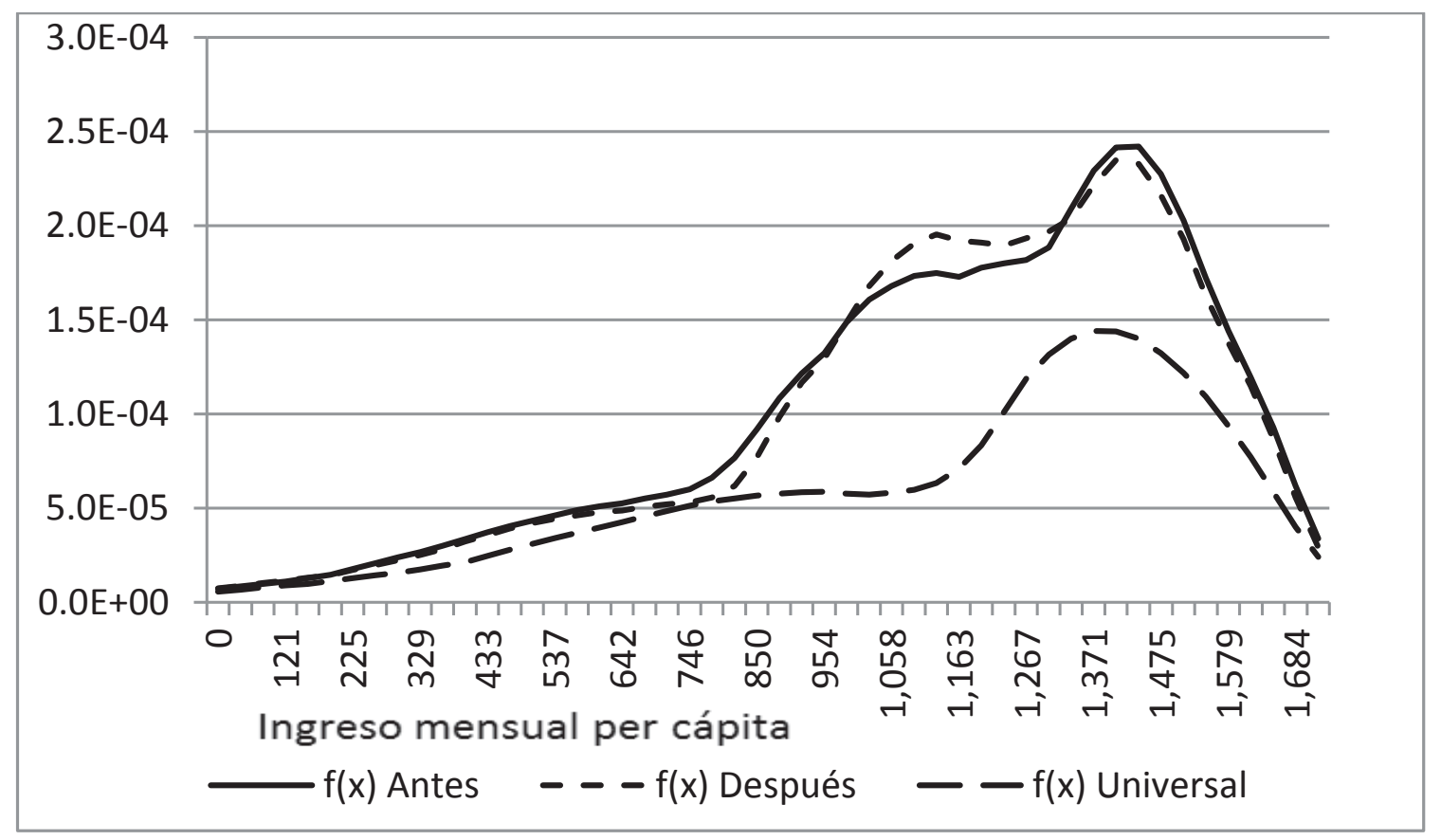


Lo anterior puede ser confirmado por el posicionamiento de la curva de densidad de la pobreza por debajo de la densidad inicial. En Baja California (figura 4), las funciones de densidad patrimonial de la pobreza se localizan más hacia la derecha con respecto de las de México, señal de que en Baja California los pobres tienen menor requerimiento de ingreso para dejar de serlo y que la entidad tiene más bajos niveles de pobreza.

El error de focalización del programa se observa en el rango de 970 hasta 1330 pesos, lo que significa que se excluye del apoyo a un número importante de hogares en situación de pobreza por patrimonio, ya que la densidad ex-post de pobreza se ubica por encima de la inicial. Por otra parte, al suponer que se transfiriere de manera universal el monto máximo estipulado en el programa Oportunidades a todo hogar pobre, fácilmente se observa una disminución significativa en la pobreza total o patrimonial -línea inferior segmentada de la figura 4.

\section{La incidencia por tipo de pobreza e impacto del programa Oportunidades}

En términos de la incidencia o porcentajes por tipo de pobreza, el cuadro 2 muestra que sin Oportunidades y con Oportunidades a nivel país los tres tipos de pobreza disminuyeron durante el periodo analizado, por lo que para el año 2006 sólo $12.76 \%$ de los hogares mexicanos padecía pobreza alimentaria, en pobreza de capacidades lo estaba $18.48 \%$ y la pobreza de patrimonio alcanzó 38.36\%. En Baja California es evidente que los porcentajes por tipo de pobreza son mucho más bajos a los observados en el país, aunque las estimaciones sin Oportunidades y con Oportunidades muestran un incremento de los hogares en pobreza alimentaria (2.92\%) y de patrimonio (17.40\%) para el año 2006; mientras que los hogares bajacalifornianos en condición de pobreza de capacidades se redujeron a 4.13\% en el periodo 2002-2006.

En relación con el impacto del programa Oportunidades por tipo de pobreza, la microsimulación de la política social Sin Oportunidades a inicios del siglo Xxi (cuadro 2, columnas 2-a $-4^{-}$) muestra que los hogares en pobreza alimentaria hubieran disminuido $28.8 \%$ a nivel nacional, pero en 


\section{Cuadro 2. México y Baja California. Impacto del programa Oportunidades por tipo de Pobreza-FGT (0), 2002-2006}

\begin{tabular}{|c|c|c|c|c|c|c|c|c|}
\hline \multirow{2}{*}{ Entidad } & \multicolumn{2}{|c|}{ Sin Oportunidades } & \multirow{2}{*}{$\begin{array}{c}\text { Variación (\%) } \\
2002-2006 \\
\end{array}$} & \multicolumn{2}{|c|}{ Con Oportunidades } & \multirow{2}{*}{$\begin{array}{c}\text { Variación (\%) } \\
2002-2006\end{array}$} & \multicolumn{2}{|c|}{ Con monto máximo ${ }^{d}$} \\
\hline & 2002 & 2006 & & 2002 & 2006 & & 2002 & 2006 \\
\hline \multicolumn{9}{|c|}{ Impacto en Pobreza Alimentaria: ${ }^{a}$} \\
\hline \multirow[t]{2}{*}{ México } & 19.14 & 13.63 & -28.8 & 18.46 & 12.76 & -30.9 & 4.31 & 2.69 \\
\hline & {$[0.004]$} & {$[0.003]$} & & {$[0.004]$} & {$[0.003]$} & & [0.002] & {$[0.001]$} \\
\hline \multirow[t]{2}{*}{ Baja California } & 1.67 & 3.05 & 82.4 & 1.67 & 2.92 & 75.1 & 0.35 & 0.35 \\
\hline & {$[0.005]$} & {$[0.007]$} & & {$[0.005]$} & {$[0.007]$} & & {$[0.003]$} & {$[0.002]$} \\
\hline \multicolumn{9}{|c|}{ *Variación respecto a la situación sin Oportunidades: } \\
\hline México & & & & & -2.09 & & & \\
\hline Baja California & & & & & -7.36 & & & \\
\hline \multicolumn{9}{|c|}{ Impacto en Pobreza de Capacidades: ${ }^{\mathrm{b}}$} \\
\hline \multirow[t]{2}{*}{ México } & 25.45 & 19.35 & -24.0 & 24.72 & 18.48 & -25.2 & 9.54 & 6.21 \\
\hline & {$[0.005]$} & [0.004] & & {$[0.005]$} & {$[0.004]$} & & [0.003] & [0.002] \\
\hline \multirow[t]{2}{*}{ Baja California } & 5.06 & 4.87 & -3.8 & 5.06 & 4.13 & -18.4 & 0.35 & 1.23 \\
\hline & {$[0.012]$} & {$[0.009]$} & & [0.012] & [0.008] & & [0.002] & [0.004] \\
\hline \multicolumn{9}{|c|}{ *Variación respecto a la situación sin Oportunidades: } \\
\hline México & & & & & -1.27 & & & \\
\hline Baja California & & & & & -14.64 & & & \\
\hline \multicolumn{9}{|c|}{ Impacto en Pobreza de Patrimonio:c } \\
\hline \multirow[t]{2}{*}{ México } & 45.59 & 38.69 & -15.1 & 45.45 & 38.36 & -15.6 & 31.27 & 24.30 \\
\hline & {$[0.006]$} & {$[0.005]$} & & {$[0.006]$} & {$[0.005]$} & & {$[0.005]$} & {$[0.004]$} \\
\hline \multirow[t]{2}{*}{ Baja California } & 14.28 & 17.62 & 23.4 & 14.28 & 17.40 & 21.8 & 7.97 & 7.28 \\
\hline & {$[0.019]$} & {$[0.017]$} & & [0.019] & [0.017] & & {$[0.015]$} & [0.011] \\
\hline \multicolumn{9}{|c|}{ *Variación respecto a la situación sin Oportunidades: } \\
\hline México & & & & & -0.46 & & & \\
\hline Baja California & & & & & -1.56 & & & \\
\hline
\end{tabular}

Nota. La cifra que aparece entre corchetes es el dato del error estándar.

${ }^{a}$ La pobreza alimentaria se refiere a los hogares cuyo ingreso per cápita es menor al necesario para cubrir las necesidades de alimentación correspondientes a los requerimientos establecidos en la canasta alimentaria INEGI-CEPAL.

${ }^{\text {b }}$ Se refiere a los hogares cuyo ingreso per cápita es menor al necesario para cubrir el patrón de consumo básico de alimentación, salud y educación.

${ }^{\mathrm{c}}$ Se refiere a los hogares cuyo ingreso per cápita es menor al necesario para cubrir el patrón de consumo básico de alimentación, vestido y calzado, vivienda, salud, transporte público y educación. ${ }^{\text {d }}$ Según el programa Oportunidades en el año 2002 el monto máximo mensual que se podría asignar a una familia eran $\$ 1550.00$ pesos $(1.29 \mathrm{SMM})$, es decir $\$ 378.05$ por persona mensual; mientras que, en el 2006 este monto eran $\$ 1855.00$ pesos (1.31 S.M.), lo que significaba $\$ 475.64$ pesos por persona al mes.

Fuente: Cálculos propios con base en la metodología del стмP, el método de Líneas de Pobreza, Micro-simulación Estática y las ENIGH 2002 y 2006. 
Baja California este tipo de pobreza se incrementaría ${ }^{16}$ en $82.4 \%$ durante el periodo 2002-2006. Una vez aplicado el programa Oportunidades (columnas $5^{\underline{a}-7}$ - ), se ve que $30.9 \%$ de los hogares que padecían pobreza alimentaria superó tal condición en el país, pero en el estado se incrementaron en $75 \%$ para el 2006; no obstante, al comparar estas variaciones porcentuales con la situación sin Oportunidades, se aprecia que los hogares en pobreza alimentaria disminuyeron apenas $2.09 \%$ en México, mientras que el programa Oportunidades evitó que este tipo de pobreza se incrementara en $7.36 \%$ más a escala estatal.

La microsimulación sin Oportunidades (cuadro 2, columnas $2^{\mathrm{a}}-4^{\mathrm{a}}$ ), permite ver que en el periodo los hogares en pobreza de capacidades hubieran disminuido $24.0 \%$ en el país y $3.8 \%$ en Baja California. Cuando se aplica el programa Oportunidades (columnas $5^{-\mathbf{a}}-7 \underline{\mathbf{a}}$ ), $25.2 \%$ de las familias que vivían en pobreza de capacidades superó este tipo de pobreza en México y $18.4 \%$ en Baja California para el 2006; pero al comparar las variaciones porcentuales, se aprecia que los hogares en pobreza de capacidades disminuyeron apenas en $1.27 \%$ en México y $14.64 \%$ en el estado de Baja California a razón del programa Oportunidades.

La microsimulación de la política social sin Oportunidades (cuadro 2, columnas $\left.2_{-}^{-} 4^{\underline{a}}\right)$ permite ver que los hogares en pobreza de patrimonio hubieran disminuido $15.1 \%$ en el país, pero en Baja California se incrementarían 23.4\% durante 2002-2006; mientras que al aplicarse el programa (columnas $5^{-}-7^{a}$ ), se advierte que $15.6 \%$ abandonó la pobreza de patrimonio a nivel nacional, pero los hogares bajacalifornianos que vivían en pobreza patrimonial ${ }^{17}$ se incrementaron en $21.8 \%$ para 2006 . La comparación de las variaciones porcentuales muestra que los hogares en pobreza patrimonial disminuyeron apenas en $0.46 \%$ en México y en Baja California el programa Oportunidades evitó que este tipo de pobreza se incrementara en $1.56 \%$ más.

En otras palabras, lo anteriormente descrito significa que en realidad la disminución de los tres tipos de pobreza no es significativa en el

${ }^{16}$ Es importante señalar que como la incidencia de pobreza alimentaria en la entidad es muy baja, los cambios en ésta se reflejan en variaciones porcentuales muy altas.

${ }^{17}$ Esto sin duda refleja lo que se ha identificado como el empobrecimiento de la clase media en la frontera norte de México y en el país; véase a Camberos (2011) y Huesca (2003). 
país a razón del programa Oportunidades. En el estado de Baja California el programa coadyuvó a que la pobreza de capacidades tuviera una ligera disminución cercana a un punto porcentual, pero la pobreza alimentaria y patrimonial se incrementó a pesar de la aplicación de Oportunidades.

Por otra parte, la microsimulación de la política social que transfiere el monto máximo a todos los hogares en pobreza muestra que esta política sería suficiente para reducir de manera significativa los tres tipos de pobreza en el país y la entidad. De haberse implementado esta política (columnas 8a-9 ), sólo 2.69\% de los hogares mexicanos hubiera padecido pobreza alimentaria y $0.35 \%$ en Baja California para el 2006; mientras que en el país $6.21 \%$ de los hogares estaría en pobreza de capacidades y en Baja California sólo 1.23\% caería en este tipo de pobreza. En México, $24.30 \%$ de los hogares sería afectado por la pobreza de patrimonio y en Baja California 7.28\% de los hogares estaría en esta situación.

En resumen, los resultados descritos denotan un modesto impacto del programa Oportunidades en la reducción de la pobreza en México y en el estado de Baja California, a la vez que dan cuenta de los errores que entraña la focalización, lo que evidentemente se hace patente en la puesta en práctica del programa Oportunidades en el país. ${ }^{18}$ Además, esta evidencia empírica pone en duda uno de los principales atributos que se le adjudican al programa Oportunidades: potenciar las capacidades de las personas en los hogares pobres, coadyuvando así a que los individuos puedan salir adelante a partir de su propio esfuerzo (Sedesol, 2003).

\section{Los costos de la pobreza y la insuficiencia de recursos asignados}

Un tercer objetivo en este estudio tiene que ver con la necesidad de conocer los recursos económicos que la sociedad requiere para afrontar y combatir el flagelo de la pobreza en los años que transcurren de la primera

${ }^{18}$ Véase la comparecencia del 15 de julio de 2008 del médico Salvador Escobedo Zoletto, ex Coordinador Nacional del Programa Oportunidades ante la Comisión de Desarrollo Social en la Cámara de Diputados, en la que se expresa este tipo de problemática. 
década en el siglo xxi. Por ello se estiman los costos para combatir la pobreza con la expresión: $C P=(q)(I)(Z)$, donde $C P$ son los costos económicos o recursos requeridos para que los hogares puedan superar la condición de pobreza; $q$ es la cantidad de hogares en pobreza, $I^{19}$ es la brecha de pobreza estandarizada y $Z$ la línea de pobreza según tipo de pobreza.

En el cuadro 3 (columnas $2^{\underline{a}-} 3^{\underline{a}}$ ) se muestra el monto de transferencias ejercido mediante el programa Oportunidades en México y la proporción asignada a Baja California por la Sedesol en el 2006. El monto total asignado al programa Oportunidades fue de casi 33 mil millones de pesos a escala nacional —equivalentes a $0.40 \%$ del producto interno bruto (PIB) de México-, mientras que para Baja California se asignaron 109.9 millones de pesos, equivalentes a $0.04 \%$ del pIB estatal.

Es necesario identificar si la magnitud de recursos destinados por la Sedesol es suficiente según las necesidades de ingreso que demanda el universo de hogares pobres de acuerdo con su número y tipo de pobreza que padecen. Las estimaciones indican que los recursos requeridos para el combate de la pobreza en el país ascendían a 168142 millones de pesos (2.05\% del PIв nacional), cinco veces más de lo que se asignó al programa Oportunidades; mientras que la estimación de costos económicos para el combate de la pobreza en Baja California muestra que el estado requería 613.5 millones de pesos y apenas recibió 109.9 millones.

En resumen, el análisis de los costos o recursos económicos requeridos para el combate a la pobreza en México y en Baja California - una vez aplicado el programa Oportunidades - permite ver que en buena medida es la insuficiencia de los recursos asignados al programa lo que viene a explicar el exiguo impacto del mismo a escala nacional y en Baja California; aunado a ello, se deben considerar los problemas que entraña propiamente el ejercicio de focalización en la política social, como lo son la inclusión de hogares que no son pobres y la exclusión de hogares que si están en pobreza.

${ }^{19}$ La familia de índices FGT calcula la incidencia o porcentaje de hogares en pobreza " $H$ " cuando el ponderador a $=0$, la intensidad de la pobreza " $I$ " medida como la distancia entre el ingreso promedio de los hogares pobres y las líneas de pobreza si $\mathrm{a}=1$ y permite incorporar la información del déficit de ingreso de los hogares más pobres cuando a $=2$; por lo tanto, atendiendo a la fórmula de los índices FGT, en este trabajo la variable $I$ se estima a partir del ponderador a $=1$ (Sen, 1976; Foster et al., 1984). 


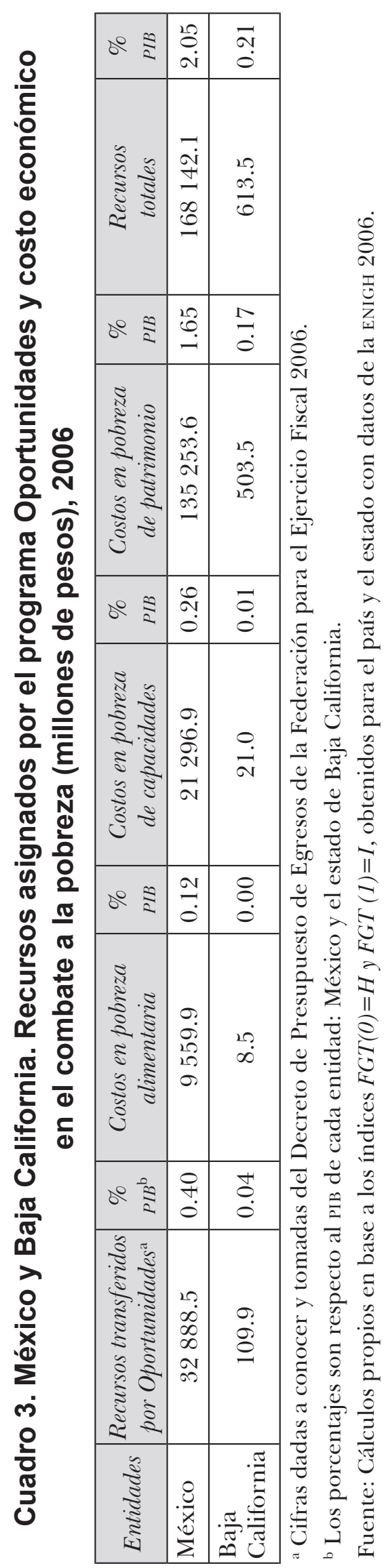




\section{Conclusiones}

La pobreza se define a partir del enfoque monetario y se estima la incidencia con el método de líneas de pobreza; luego, la técnica de microsimulación estática permite medir el efecto que tienen las transferencias monetarias Oportunidades en los niveles de pobreza de México y Baja California, variaciones de las cuales también da cuenta el análisis no paramétrico. Se encontró un impacto muy reducido del programa en el país, pues el mismo contribuyó a que los hogares en pobreza alimentaria disminuyeran apenas $2.09 \%$, las familias en pobreza de capacidades $1.27 \%$ y en pobreza patrimonial sólo $0.46 \%$.

En Baja California la incidencia de hogares en pobreza de capacidades disminuyó cerca de un punto porcentual, y pese a que Oportunidades no contrarrestó el incremento en los niveles de pobreza alimentaria y patrimonial, sí evitó que la proporción de hogares que vivían en pobreza alimentaria se incrementara en $7.36 \%$ y la proporción de hogares en pobreza de patrimonio en $1.56 \%$.

La evidencia empírica pone en duda uno de los principales atributos que se adjudican al programa Oportunidades: potenciar las capacidades de las personas en los hogares pobres para que éstas puedan salir adelante por su propio esfuerzo; es decir, esta política social en realidad no está ayudando a combatir de manera significativa la pobreza en el país ni en el estado de Baja California, lo que lleva a rechazar la hipótesis diseñada en este estudio.

El análisis de los costos o recursos requeridos para el combate a la pobreza en México y Baja California muestra que en buena medida es la insuficiencia de los recursos asignados al programa Oportunidades lo que explica el exiguo impacto del mismo a escala nacional y también en Baja California. Aunado a ello, se deben considerar los problemas que entraña propiamente el ejercicio de focalización en la política social, a saber: los errores de omisión/exclusión de hogares que están en pobreza y la inclusión de hogares que no son pobres.

Finalmente, vale la pena enfatizar el importante papel que tendría una política social focalizada que transfiriera el monto máximo a todo hogar 
pobre,${ }^{20}$ aunque evidentemente tendría que ir acompañada de una política económica generadora de crecimiento y empleo, para que el desarrollo de capacidades individuales se pueda transformar en capital humano; esto es, para que los sectores pobres se puedan insertar en el proceso productivo haciéndose de un ingreso que, a la vez que les ayude a superar la pobreza, les permita romper la dependencia respecto de las transferencias, así como una mejor inserción en la vida social.

\section{Bibliografía}

Aguilar, G.G. (2000). Desigualdad y pobreza en México, ¿sson inevitables? México: Universidad Nacional Autónoma de México-Instituto de Investigaciones Económicas, Instituto Politécnico Nacional, Centro de Investigaciones Económicas, Administrativas y Sociales.

Atkinson, A., Bourguignon, F., O'Donogui, C., Sutherland, H. y Utili, F. (2002). Microsimulation of social policy in the European Union: Case study of a European minimum pension. Economica, 69, 229-243.

Banco Mundial. (2004). La pobreza en México: Una evaluación de las condiciones, tendencias y estrategias del gobierno. México: Autor.

Bautista, S. (2004). Evaluación del efecto de Oportunidades sobre la utilización de los servicios de salud en el medio rural. (Documento de investigación, No. 9), pp. 1-66. Secretaría de Desarrollo Social.

Bourguignon, F. (2003). From income to endowments: The difficult task of expanding the income poverty paradigm (Documento de trabajo, No. 2003-03). París: Centre National de la Recherche Scientifique, Departement et Laboratoire de Economie Theorique et Appliquee-World Bank.

Bourguignon, F. y Spadaro, A. (2006). Microsimulation as a tool for evaluating redistribution policies. (Documento de trabajo, No. 20), Society for the Study of Economic Inequality.

Bourguignon, F., Ferreira, H. G. y Leite, F. (2003). Conditional cash transfers, schooling and child labor: Micro-Simulating Brasil's Bolsa Escola Program. The World Bank Economic Review, 17(2), 229-254.

Bracamontes, J. y Huesca, L. (2011). Evaluación del programa Oportunidades y su impacto en México y la frontera norte. En L. Huesca, M. Camberos y C.

${ }^{20}$ Ello implicaría un significativo incremento en los recursos asignados para el combate a la pobreza en el país y en el estado de Baja California. 
Calderón (Coords.), El bienestar y desarrollo en el siglo XXI (pp. 221-240). México: Centro de Investigación en Alimentación y Desarrollo, Plaza y Valdés.

Bracamontes, J., Camberos, M. y Huesca, L. (2011). El programa Oportunidades y el ejercicio de focalización en México. Saarbrücken, Alemania: Editorial Académica Española.

Camberos, M. (2013). El empobrecimiento de la clase media en la frontera: Saldos del neoliberalismo. En L. Huesca, C. Calderón y M. Camberos, (Coords.), El bienestar en el siglo XXI (pp. 21-36), Plaza y Valdés.

Camberos, M. y Bracamontes, J. (1996). Análisis comparativo de la pobreza en la frontera norte de México. En A. Mungaray y M. G. García de León (Coords.), Desarrollo fronterizo y globalización (pp. 107-124). México: Colegio de Economistas, Gobierno del Estado de Sonora.

Camberos, M., Huesca, L. y Genesta, M. A. (1996). Pobreza regional en Sonora: Tendencias recientes, recursos necesarios para combatirla y perspectivas para el año 2000. Investigación Económica, LVI(215), 163-200.

Cifras históricas de los apoyos. Recuperado de <www.oportunidades.gob.mx/ Portal/wb/Web/oportunidades_historico $>$.

Cogneau, D., Grimm M. y Robillird, S. (2003). Evaluating poverty reduction policies. The contribution of micro-simulation techniques. En J-P. Cling, M. Razafindrakato y F. Roubaud (Eds.). New international poverty reduction strategies (pp. 340-370). Londres: Routledge Books.

Consejo Nacional de Evaluación de la Política de Desarrollo Social (Coneval). (2006). Nota técnica sobre la aplicación de la metodología del Comité Técnico para la Medición de la Pobreza 2000-2005. Recuperado de <www.coneval. gob.mx $>$.

Cornia, G. y Stewart, F. (2003). Subsidios alimentarios y dos errores de focalización. Comercio Exterior, 53(6), 563-573.

Cortés, F., Bánegas, I. y Solís, P. (2007). Pobres con Oportunidades: México 20022005. Estudios Sociológicos, XXV(1), 3-40.

Decreto de Presupuesto de Egresos de la Federacióon. Recuperado de <http:// www.shcp.gob.mx/EGRESOS/PEF/Paginas/PresupuestodeEgresos.aspx $>$.

Demián, A. y Boltvinik, J. (2003). Evolución y características de la pobreza en México. Comercio Exterior, 53(6), 525-528.

Duclos, Jean-Yves y Abdelkrim, A. (2006). Poverty and equity. Measurement, policy, and estimation with $D A D$. Nueva York: Springer, International Development Research Centre.

Foster, J., Greer, J. y Thorbecke, E. (1984). A class of descomposable poverty measures. Econometrica, 52(3), 761-766.

González, T. E. (2002). La evaluación de los programas sociales: Aspectos conceptuales y prácticos. Economía Informa, (303), 22-27. 
Hernández, G. y Del Razo, L. M. (Octubre, 2004). Lo que dicen los pobres: Evaluación del impacto de los programas sociales sobre la percepción de los beneficiarios (Documento de investigación, No. 14). México: Secretaría de Desarrollo Social.

Huesca, R. L. (2003). Análisis de la polarización del ingreso de los hogares en México durante 1984-2000. Investigación Económica, LXII(246), 89-123.

Instituto Nacional de Estadística, Geografía e Informática (INEGI). (2002). Encuesta Nacional de Ingresos y Gastos de los Hogares. México: Autor.

Instituto Nacional de Estadística, Geografía e Informática (INEGI). (2006). Encuesta Nacional de Ingresos y Gastos de los Hogares. México: Autor.

Instituto Nacional de Estadística, Geografía e Informática (INEGI) y Comisión Económica para América Latina y el Caribe (CEPAL). (1993). Magnitud y evolución de la pobreza en México, 1984-1992. Aguascalientes, México: Organización de las Naciones Unidas, CEPAL-INEGI.

Jantty, M. y Danziger, S. (2000). Income poverty in advanced countries. En A. B. Atkinson y F. Bourguignon (Eds.), Handbook of income distribution (volumen I, pp. 309-379). Amsterdam: Elsevier Science.

Jenkins, S. y Van, P. (2005). Accounting for income distribution trends: A density function decomposition approach. Journal of Economic Inequality, (3), 43-62.

Kanbur, R. (2002). Conceptual challenges in poverty and inequality: One development economist's perspective (Documento de trabajo, No. 2002-09). Recuperado del sitio de internet de Cornell University: <http://aem.cornell.edu/research/ wp.htm\#wp2002>.

Kanbur, R. y Squire, L. (2001). The evolution of thinking about poverty: Exploring the interactions. En G. Meier y J. Stiglitz (Eds.), Frontiers of development economics: The future in perspective (pp. 183-126). Reino Unido: Oxford University Press.

Ocho metas del milenio. Recuperado de <www.un.org/es/millenniumgoals/ $>$.

Parker, S. (Noviembre, 2003). Evaluación del impacto de Oportunidades sobre la inscripción escolar: Primaria, secundaria y media superior (Documento de investigación, No. 6). México: Secretaría de Desarrollo Social.

Perry, G., Arias, O. S., López, J. H., Maloney, W. F. y Servén, L. (2006). Poverty reduction and growth: Virtuos and vivious circles. Washington D.C: World Bank, Banco Internacional de Desarrollo.

Ruggeri, C., Saith, R. y Stewart, F. (Mayo, 2003). Does it matter that we do not agree on the definition of poverty? A comparison of four approaches (Documento de trabajo, No. 107). Queen Elizabeth House, University of Oxford.

Secretaría de Desarrollo Social (Sedesol). (2003). Programa institucional Oportunidades 2002-2006. México: Autor.

Secretaría de Desarrollo Social (Sedesol)-Comité Técnico para la Medición de la Pobreza (СтPM). (Junio, 2002). Medición de la pobreza, variantes metodo- 
lógicas y estimación preliminar (Documento de investigación, No. 1). México: Autor.

Sen, A. (1976). Poverty: An ordinal approach to measurement. Econometrica, (44), 219-232.

Sen, A. (1999). Poverty as capability deprivation. En Development as freedom (pp. 87-110). Reino Unido: Oxford University Press.

Silverman, B. W. (1986). Density estimation for statistics and data analysis. Londres: Chapman and Hall.

Wand, M. P. y Jones, M. C., (1995). Kernel smoothing, Londres: Chapman and Hall. World Bank. (2005). World Development Indicators, 2004. Washington, D.C: Autor.

Zhan, Q. (2003). DAD, an innovative tool for income distribution analysis. Journal of Economic Inequality, 1(3), 281-284. 